\title{
Model for the Surface Anisotropy Field Observed in Spin-Wave Resonance in (Ga,Mn)As Thin Films
}

\author{
H. PUSZKARSKI ${ }^{a, *}$ AND P. TOMCZAK ${ }^{b}$ \\ ${ }^{a}$ Surface Physics Division, Faculty of Physics, Adam Mickiewicz University, Umultowska 85, 61-614 Poznań, Poland \\ ${ }^{b}$ Quantum Physics Division, Faculty of Physics, Adam Mickiewicz University, \\ Umultowska 85, 61-614 Poznań, Poland
}

\begin{abstract}
In this study we show why spin-wave resonance in a (Ga,Mn)As thin film may, in different conditions, meet the assumptions of either the surface inhomogeneity (SI) or the volume inhomogeneity (VI) model.
\end{abstract}

DOI: 10.12693 /APhysPolA.127.508

PACS: 75.50.Pp, 76.50.+g, 75.70.-i, 75.30.Ds

\section{Introduction}

Dilute ferromagnetic semiconductors are a class of very promising materials of the future [1-6]. Gallium manganese arsenide (Ga,Mn)As, created on the basis of the semiconductor gallium arsenide by the addition of a small percentage of manganese as a magnetic dopant, is one of the most intensively studied compounds in this class [712]. Spin-wave resonance in thin films has been studied particularly intensively in gallium manganese arsenide in the past decade [13-21]. Especially rich resonance spectra were obtained in studies with a variable configuration of the static field with respect to the film surface. The field was rotated perpendicularly to the film surface, which corresponds to variable polar angle $\theta_{H}$ between the direction of the external field and the surface normal. In the present paper we shall analyze SWR measurement data concerning the out-of-plane rotation of the magnetic field, mainly because of the controversy that arose in the interpretation of these results over an issue which therefore requires elucidation. If researchers tend to agree on the interpretation of SWR spectra in two extreme configurations - the perpendicular and parallel configurations, corresponding to $\theta_{H}=0$ and $\theta_{H}=90^{\circ}$, respectively - the interpretation of results obtained in intermediate configurations is under debate. Almost as a rule, a particular configuration of the external field tends to occur in this range at a critical angle $\theta_{H}^{c}$, for which the multi-peak SWR spectrum collapses to a single-peak FMR spectrum. There are two schools of thought regarding the interpretation of the occurrence of this critical angle. Both schools agree that in the critical configuration the thin film is magnetically homogeneous, and the boundary conditions (specifically, the surface spin pinning) correspond to the natural conditions, only resulting from the reduced neighborhood of the surface

*corresponding author; e-mail: henpusz@amu.edu.pl spins. The difference of opinion concerns the configuration evolution leading to the above-described "naturally homogeneous" magnetic state. One school [18] uses the surface inhomogeneity (SI) model and assumes that rotation of the magnetic field does not modify the profile of the bulk magnon potential, which remains homogeneous across the film; only the surface pinning conditions change, diverging from the natural conditions as the angle grows above or decreases below the critical configuration (with the surface pinning decreasing or increasing). In contrast, the other school [20, 21], using the volume inhomogeneity (VI) model, claims that it is the bulk profile of the magnon potential that changes with the field configuration: remaining linear, but inclined at different angles with respect to the surface of the film, the magnon potential increases or decreases inside the film as the configuration diverges from the critical angle, while the natural conditions prevail invariably on the surface. In this paper we opt for the interpretation based on the SI model and propose a theoretical model of the configuration evolution of the surface spin pinning in agreement with the experimental data. Our interpretation leads to some physical conclusions, which provide new insights into the surface properties of ferromagnetic semiconductor (Ga,Mn)As thin films.

\section{Out-of-plane angle dependence of the surface parameter in (Ga,Mn)As thin films}

The experimental SWR spectra analyzed in this paper were measured in the "out-of-plane geometry", as referred to by the Authors of Ref [18]. The external magnetic field $\boldsymbol{H}$ was confined to the horizontal plane (i.e. perpendicular to the film surface) allowing SWR measurements with $\boldsymbol{H}$ in any intermediate orientation between the normal to the film surface, $\boldsymbol{H} \|[001]$, and the in-plane orientation, $\boldsymbol{H} \|[1 \overline{1} 0]$. In this particular geometry the magnetization $\boldsymbol{M}$ of the sample lies in the same horizontal plane as the field $\boldsymbol{H}$. Thus, the spatial orientation of the vectors $\boldsymbol{H}$ and $\boldsymbol{M}$ is defined by two polar angles, $\theta_{H}$ and $\theta_{M}$, between the respective vectors and the normal to the surface of the film. 


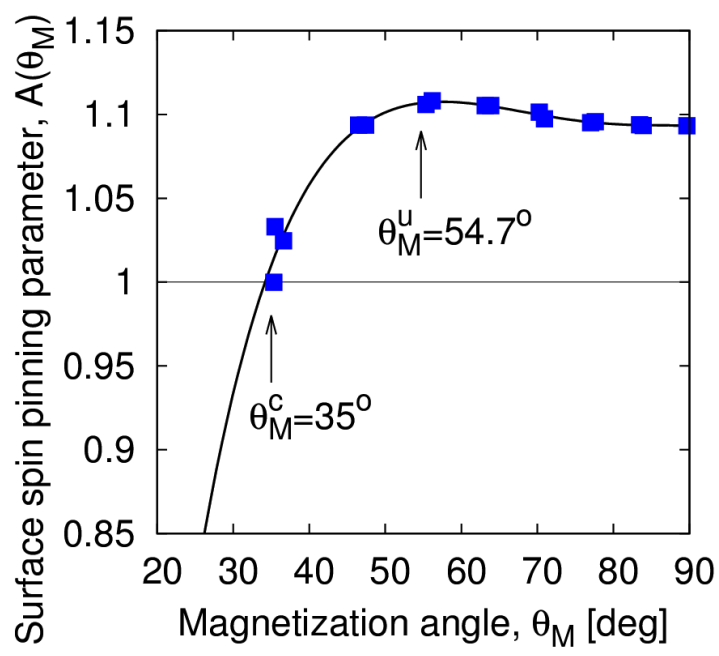

Fig. 1. Magnetization angle dependence of the surface pinning parameter $A\left(\theta_{M}\right)$ according to the experimental data obtained by Liu et al [18].

On the basis of their SWR study of (Ga,Mn)As thin films Liu et al [18]. plotted the configuration dependence of the surface parameter $A\left(\theta_{H}\right)$ with the magnetic field rotating from the perpendicular $\left(\theta_{H}=0\right)$ to parallel $\left(\theta_{H}=90^{\circ}\right)$ configuration. Figure 1 shows the configuration dependence of the surface parameter, which we have recalculated to plot $A$ versus the new variable $\theta_{M}$; the graph corresponds to the measurement data of Liu et al. presented in Ref [18], Fig. 9. The natural surface pinning is found to occur for the critical angle $\theta_{M}=35^{\circ}$ (which corresponds to the experimental angle $\theta_{H}=19^{\circ}$ ). Also, the new plot reveals the occurrence of a local maximum in the $A\left(\theta_{M}\right)$ dependence around the angle $\theta_{M}^{u}=54.73^{\circ}$, for which the term $\left(3 \cos ^{2} \theta_{M}^{u}-1\right)$ equals zero (below we shall take advantage of this finding in further analysis of the experimental data obtained by Liu et al [18]).

Note that the experiment only provided the values of the surface parameter $A$ in the $\theta_{M}$ range in which $A \geq 1$; in the angle range corresponding to $A<1$ the solid line in Fig. 1 is a mere extrapolation of the dependence beyond this range. Strikingly, two characteristic angles can be observed in the $A\left(\theta_{M}\right)$ dependence, one-the critical angle $\theta_{M}^{c}$-corresponding to $A\left(\theta_{M}^{c}\right)=1$, the other-which we will call the magic angle $\theta_{M}^{u}$-to a local maximum $A\left(\theta_{M}^{u}\right)=\max$. The angular dependence of the surface parameter presented in Fig. 1 can be described by the function:

$$
A\left(\theta_{M}\right)=1+a_{0}-a_{2}\left(\theta_{M}\right)\left(3 \cos ^{2} \theta_{M}-1\right) .
$$

Thus, the following equalities are fulfilled at the abovementioned characteristic angles:

$$
a_{0}=a_{2}\left(\theta_{M}^{c}\right)\left(3 \cos ^{2} \theta_{M}^{c}-1\right)
$$

for the critical angle $\theta_{M}^{c}$, and:

$$
3 \cos ^{2} \theta_{M}^{u}-1=0
$$

for the magic angle $\theta_{M}^{u}$ corresponding to the maximum value $A_{\max }\left(\theta_{M}^{u}\right)=1+a_{0}$. The procedure of numerical fitting of the relation (1) to the measurement points shown in Fig. 1 allows to determine the function $a_{2}\left(\theta_{M}\right)$. On the other hand, according to our earlier considerations in Refs. [22-24], $a_{2}\left(\theta_{M}\right)$ can be expressed as follows by structural and magnetic quantities:

$$
a_{2}\left(\theta_{M}\right)=\frac{1}{2}\left[4 \pi\left(M_{\mathrm{eff}}^{\mathrm{bulk}}-M_{\mathrm{eff}}^{\text {surface }}\right) \frac{a^{2}}{D_{\mathrm{ex}}}\right],
$$

where $4 \pi M_{\text {eff }} \equiv 4 \pi M-H_{2} \perp, M$ is the saturation magnetization, $H_{2} \perp$ the effective uniaxial anisotropy field, $a$ the lattice constant (the average $\mathrm{Mn}-\mathrm{Mn}$ distance), and $D_{\text {ex }}$ the exchange stiffness constant.

\section{Physical implications of the model}

Using the formula (4) and knowing the $a_{2}\left(\theta_{M}\right)$ dependence (determined numerically), we could determine the configuration dependence (see Fig. 2) of the quantity defined:

$$
\begin{aligned}
& 4 \pi \Delta M_{\mathrm{eff}}=4 \pi M_{\mathrm{eff}}^{\text {bulk }}-4 \pi M_{\mathrm{eff}}^{\text {surface }} \equiv \\
& 4 \pi\left(M^{\text {bulk }}-M^{\text {surface }}\right)-\left(H_{2 \perp}^{\text {bulk }}-H_{2 \perp}^{\text {surface }}\right),
\end{aligned}
$$

which relates the bulk and surface effective magnetizations. From Fig. 2 it follows that the magic angle is that particular angle at which the two (bulk and surface) effective magnetizations become equal.

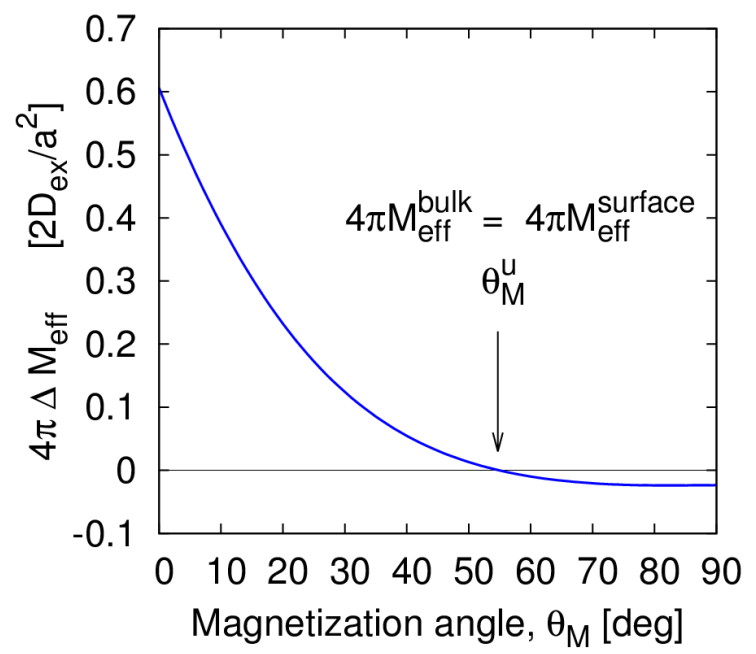

Fig. 2. Magnetization angle dependence of the surface contribution to the effective bulk magnetization $4 \pi M_{\mathrm{eff}}^{\text {bulk }}$ calculated from Eq. (4) (in units of $2 D_{\mathrm{ex}} / a^{2}$ ); $4 \pi \Delta M_{\text {eff }}$ is defined by Eq. (5).

This is a consequence of the following interpretation of Fig. 2: When the field $\boldsymbol{H}$ is perpendicular to the surface of the film (which corresponds to $\theta_{M}=0$ ), both the surface and bulk magnetizations can be assumed to be aligned with the out-of-plane easy axis normal to the surface of the film, but the bulk magnetization predominates over the surface magnetization, since, as indicated by Fig. 2, $4 \pi \Delta M_{\text {eff }}>0$ for $\theta_{M}=0$. As soon as the orientation of the applied field diverges from the normal direction, all the spins immediately incline towards the film plane, in which the (Ga,Mn)As film has two easy 
axes; this will result in reduced effective bulk magnetization. The surface magnetization, however, will not decrease as much as the bulk magnetization, since the surface spins are additionally constrained by the uniaxial surface anisotropy field that aligns them in the direction normal to the surface of the film. Thus, $M^{\text {bulk }}$ will decrease at a higher rate than $M^{\text {surface }}$ as the field will continue to rotate towards the film plane; as a consequence, the two effective magnetizations will be approaching a common value to reach it at the magic angle $\theta_{M}^{u}$.

The particular role of the magic angle can be also demonstrated otherwise, by referring to the interaction length $\lambda$ defined separately in the bulk and surface (and denoted $\lambda_{b}$ and $\lambda_{s}$, respectively):

$$
\lambda_{b}=\sqrt{\frac{D_{\mathrm{ex}}}{4 \pi M_{\mathrm{eff}}^{\text {bulk }}}}, \quad \lambda_{s}=\sqrt{\frac{D_{\mathrm{ex}}}{4 \pi M_{\mathrm{eff}}^{\text {surface }}}} .
$$

At the magic angle the two interaction lengths defined above are equal. In the angle range $\theta_{M}>\theta_{M}^{u}$ the surface interaction length $\lambda_{s}$ is shorter than the bulk interaction length $\lambda_{b}$; for $\theta_{M}<\theta_{M}^{u}$, in contrast, $\lambda_{s}>\lambda_{b}$. The model using the concept of local interaction length provides an intuitive explanation of an experimental finding [18] that has not been elucidated yet, that spin-wave resonance in $(\mathrm{Ga}, \mathrm{Mn})$ As thin films meets the assumptions of the surface inhomogeneity (SI) model [25-28] in the in-plane configuration of the external field, and the volume inhomogeneity (VI) model [29] in the out-of-plane configuration with the field oriented close to the surface normal.

The plot shown in Fig. 2 indicates that the surface interaction length $\lambda_{s}$ is only slightly smaller than the bulk exchange length $\lambda_{b}$ for any angle $\theta_{M}$ between the magic angle $\theta_{M}^{u}$ and the parallel configuration angle $\theta_{M}=90^{\circ}$; thus, in this angle range a surface disturbance will not go beyond the first sub-surface plane formed by the spins directly under the surface. This means that the assumptions of the SI model are fulfilled very well in this angle range. In contrast, for angles $\theta_{M}<\theta_{M}^{u} \lambda_{s}$ is greater than $\lambda_{b}$ and grows steeply as the perpendicular configuration $\theta_{M}=0$ is approached. This means that in this angle range a surface disturbance will not be localized at the surface, but rather will penetrate into the bulk, affecting deeper sub-surface planes. Thus, the applicability of the SI model is very limited in this angle range, and the volume inhomogeneity model will be more adequate.

\section{Acknowledgments}

This study was supported by NCN (National Science Centre of Poland), Grant no. DEC2013/08/M/ST3/00967.

\section{References}

[1] J.K. Furdyna, J. Appl. Phys. 64, R29 (1988).

[2] H. Ohno, Science 281, 951 (1998).

[3] T. Dietl, H. Ohno, F. Matsukura, J. Cibert, D. Ferrand, Science 287, 1019 (2000).

[4] T. Jungwirth, Jairo Sinova, J. Mašek, J. Kučera, A.H. MacDonald, Rev. Mod. Phys. 78, 809 (2006).
[5] K. Sato, L. Bergqvist, J. Kudrnovský, P.H. Dederichs, O. Eriksson, I. Turek, B. Sanyal, G. Bouzerar, H. Katayama-Yoshida, V.A. Dinh, T. Fukushima, H. Kizaki, R. Zeller, Rev. Mod. Phys. 82, 1633 (2010).

[6] T. Dietl, Nat. Mater. 9, 965 (2010).

[7] M. Sawicki, F. Matsukura, T. Dietl, G.M. Schott, C. Ruester, G. Schmidt, L.W. Molenkamp, G. Karczewski, J. Supercond. Novel Magn. 16, 7 (2003).

[8] M. Sawicki, K.-Y. Wang, K. W. Edmonds, R.P. Campion, C.R. Staddon, N.R.S. Farley, C.T. Foxon, E. Papis, E. Kamińska, A. Piotrowska, T. Dietl, B.L. Gallagher, Phys. Rev. B 71, 121302(R) (2005).

[9] X. Liu, J.K. Furdyna, J. Phys.: Condens. Matter 18, R245 (2006).

[10] G. Bouzerar, Europhys. Lett. 79, 57007 (2007).

[11] A. Werpachowska, T. Dietl, Phys. Rev. B 82, 085204 (2010).

[12] P. Němec, V. Novák, N. Tesařová, E. Rozkotová, H. Reichlová, D. Butkovičová, F. Trojánek, K. Olejník, P. Malý, R.P. Campion, B.L. Gallagher, Jairo Sinova, T. Jungwirth, Nat. Commun. 4, 1422 (2012).

[13] Y. Sasaki, X. Liu, T. Wojtowicz, J.K. Furdyna, J. Supercond. Novel Magn. 16, 143 (2003).

[14] X. Liu, Y. Sasaki, J.K. Furdyna, Phys. Rev. B 67, 205204 (2003).

[15] T.G. Rappoport, P. Redliński, X. Liu, G. Zaránd, J.K. Furdyna, B. Jankó, Phys. Rev. B 69, 125213 (2004).

[16] X. Liu, W. L. Lim, M. Dobrowolska, J.K. Furdyna, T. Wojtowicz, Phys. Rev. B 71, 035307 (2005).

[17] X. Liu, J.K. Furdyna, J. Phys.: Condens. Matter 18, R245 (2006).

[18] X. Liu, Y.-Y. Zhou, J.K. Furdyna, Phys. Rev. B 75 , 195220 (2007).

[19] S.T.B. Goennenwein, T. Graf, T. Wassner, M.S. Brandt, M. Stutzmann, J.B. Philipp, R. Gross, M. Krieger, K. Zürn, P. Ziemann, A. Koeder, S. Frank, W. Schoch, A. Waag, Appl. Phys. Lett. 82, 730 (2003).

[20] C. Bihler, W. Schoch, W. Limmer, S.T.B. Goennenwein, M.S. Brandt, Phys. Rev. B 79, 045205 (2009).

[21] L. Dreher, C. Bihler, E. Peiner, A. Waag, W. Schoch, W. Limmer, S.T.B. Goennenwein, M.S. Brandt, Phys. Rev. B 87, 224422 (2013).

[22] H. Puszkarski, M. Kasperski, Acta Phys. Pol. A 121, 1165 (2012)

[23] H. Puszkarski, Solid State Comm. 22, 563 (1977).

[24] A.P. Cracknell, H. Puszkarski, Solid State Comm. 28, 891 (1978).

[25] H. Puszkarski, Acta Phys. Pol. A 38, 217\&899 (1970)

[26] J.T. Yu, R.A. Turk, P.E. Wigen, Phys. Rev. B 11 , 420 (1975).

[27] P.E. Wigen, H. Puszkarski, Solid State Comm. 18 363 (1976).

[28] H. Puszkarski, Progr. Surf. Sci. 9, 247 (1979).

[29] A.M. Portis, Appl. Phys. Lett. 2, 69 (1963). 